\title{
Study on the differential proteins in plasma of the BPD sensitive to dexamethasone therapy
}

\author{
huaiping Cheng ${ }^{1}$, Yian Tian ${ }^{2}$, Yafei Zheng ${ }^{1}$, Haiyan Zhu ${ }^{1}$, wei Wang ${ }^{1}$, Tianping Bao ${ }^{1}$, \\ Rong $\mathrm{Wu}^{3}$, and zhaofang $\operatorname{Tian}^{1}$ \\ ${ }^{1}$ The Affiliated Huaian No.1 People's Hospital of Nanjing Medical University \\ ${ }^{2}$ University of Barcelona \\ ${ }^{3}$ Huaian Maternity and Children hospital affiliated to Anhui Medical University
}

June 19, 2020

\begin{abstract}
Abstract Objective To find some plasma differential proteins in Bronchopulmonary dysplasia(BPD) sensitive to dexamethasone therapy.Methods 30 cases of BPD from NICU of the affiliated Huaian first people's hospital of Nanjing Medical University were selected,the plasma samples were collected before a 10-day course dexamethasone therapy for all objects,and the therapeutic effect was judged sooner after the therapy finished.The infants who showed significant therapeutic effect were collected the plasma samples again. Then, 3 infants were picked at random from the dexamethasone sensitive infants , whose plasma samples before and after dexamethasone therapy were analyzed by DIA, and the differential proteins were found and analyzed by bioinformatics. Results 10 of 30 cases were sensitive to dexamethasone. There are 23 differential proteins between two groups(before and after dexamethasone administration) in total, from which, 27 proteins are downregulated,while 6 upregulated. Bioinformatics analysis showed that differential proteins may involve multiple genes and signal pathways.. When combined with literature retrieval,both low-density lipoprotein receptor-related protein 1(LRP1) and S100A8 might be consindered as potential targets for dexamethasone intervention of BPD.Conclusion We found the differential proteins related to the sensitivity to glucocorticoids for $\mathrm{BPD}$, which will provide the direction for the choice of glucocorticoids in the treatment of BPD .
\end{abstract}

\section{Hosted file}

manuscript.docx available at https://authorea.com/users/335167/articles/461068-study-on-thedifferential-proteins-in-plasma-of-the-bpd-sensitive-to-dexamethasone-therapy

\section{Hosted file}

Table I(1).docx available at https://authorea.com/users/335167/articles/461068-study-on-thedifferential-proteins-in-plasma-of-the-bpd-sensitive-to-dexamethasone-therapy

\section{Hosted file}

Table2.docx available at https://authorea.com/users/335167/articles/461068-study-on-thedifferential-proteins-in-plasma-of-the-bpd-sensitive-to-dexamethasone-therapy

\section{Hosted file}

Tab 3.docx available at https://authorea.com/users/335167/articles/461068-study-on-thedifferential-proteins-in-plasma-of-the-bpd-sensitive-to-dexamethasone-therapy 


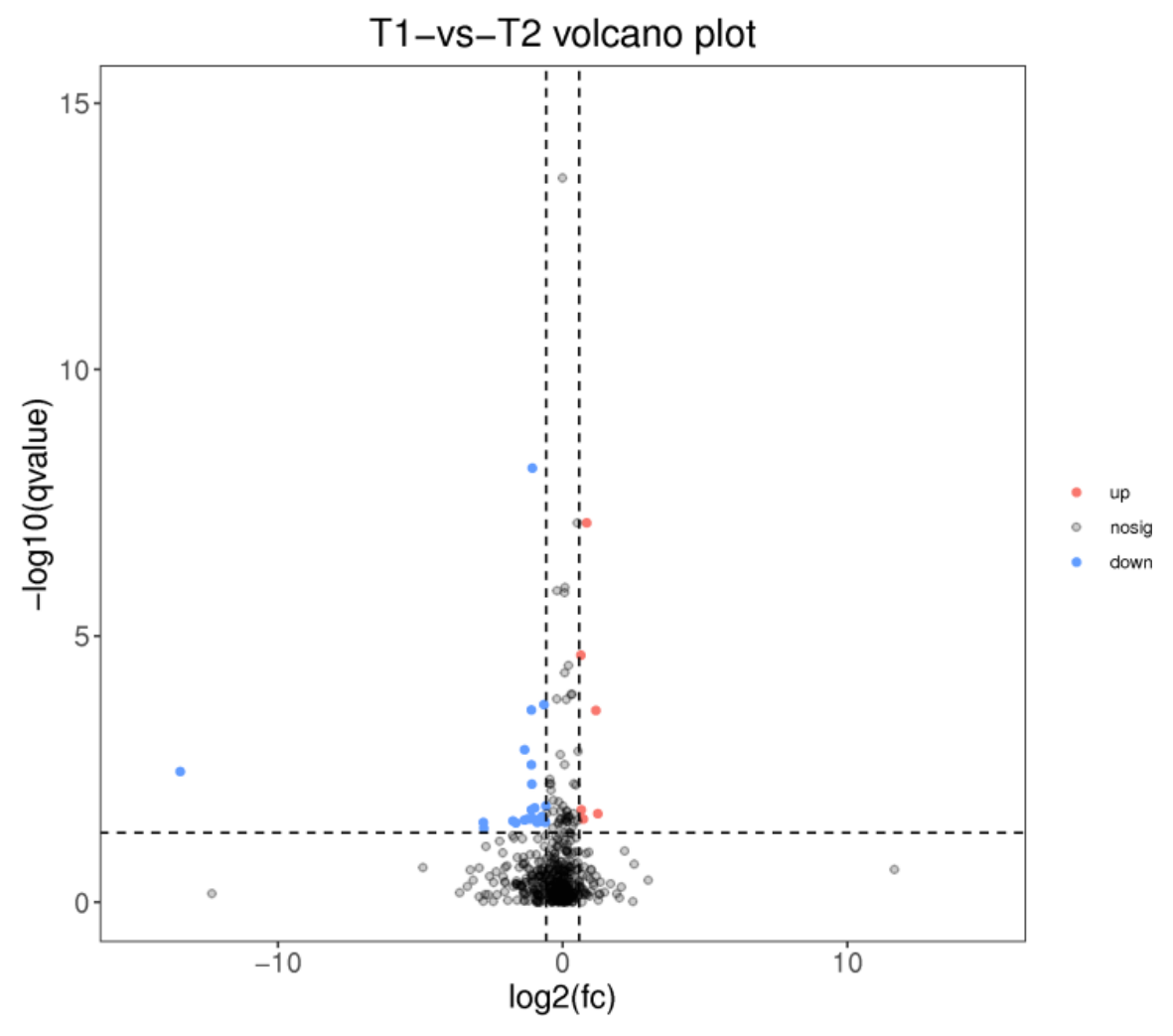




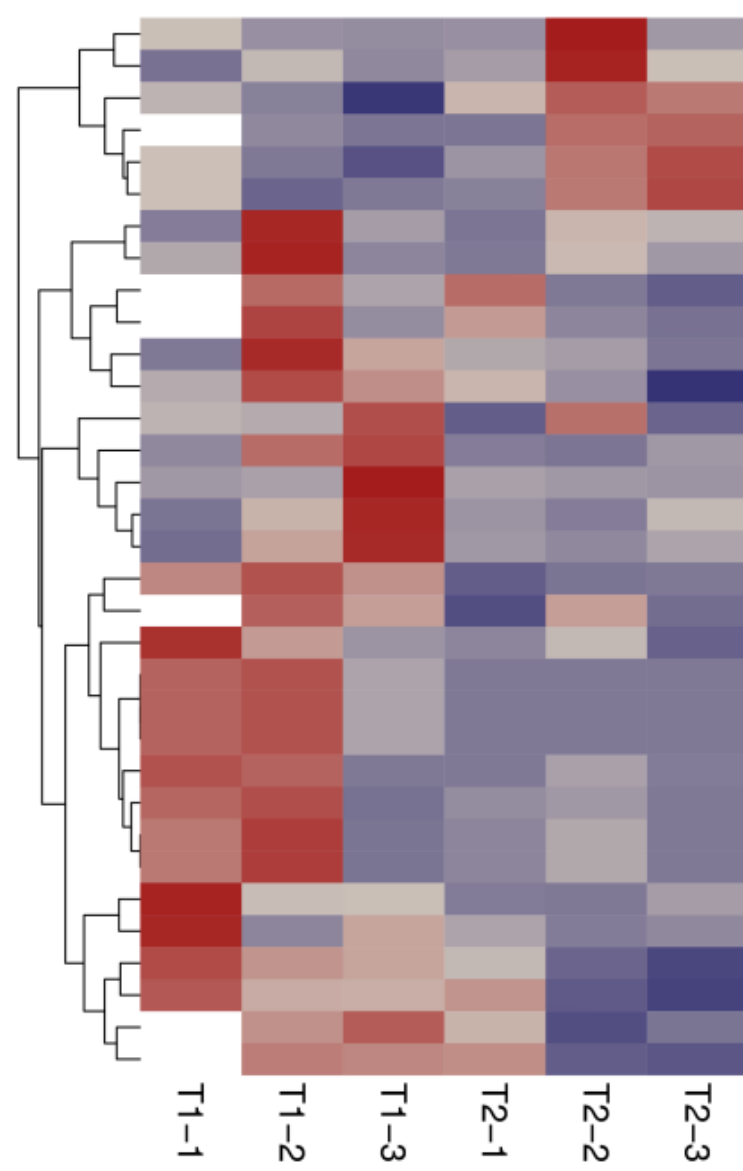

ENSG00000257017

ENSG000000542

ENSG00000175336

ENSG00000118271

ENSG00000099769

ENSG00000133742

ENSG00000244734

ENSG00000146070

ENSG00000189184

ENSG00000275395

ENSG00000129009

ENSG00000111669

ENSG00000136167

ENSG00000130635

ENSG00000143546

ENSG00000067225

ENSG00000173546

ENSG00000109971

ENSG00000111799

ENSG00000243678

ENSG00000011052

ENSG00000239672

ENSG00000167815

ENSG00000196565

ENSG00000188536

ENSG00000206172

ENSG00000134333

ENSG00000111341

ENSG00000123384

ENSG00000108821

ENSG00000161944

ENSG00000169575

$\begin{array}{llllll}\exists & \exists & \exists & \vec{N} & \vec{N} & \vec{N} \\ \vec{I} & \overrightarrow{1} & \overrightarrow{1} & 1 & 1 & 1\end{array}$

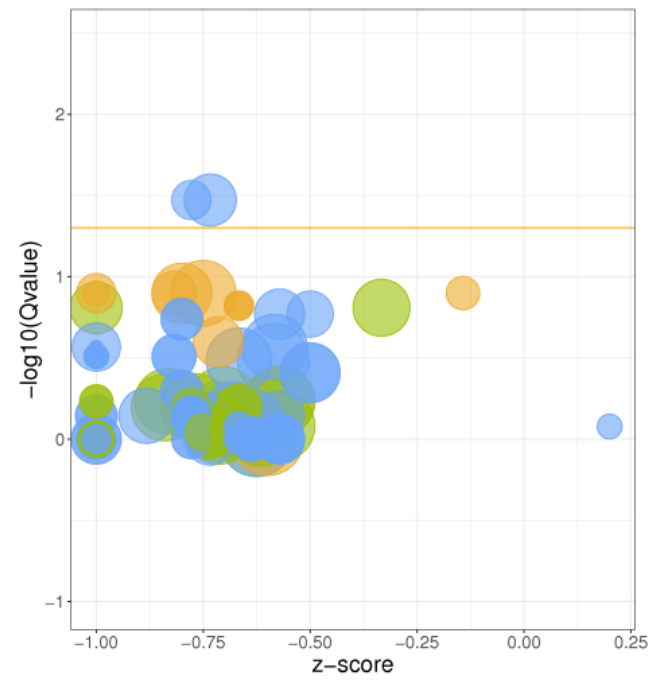

GO term Top 20

\begin{tabular}{|c|c|}
\hline ID & Descrption \\
\hline GO:0009056 & catabolic process \\
\hline GO:0051186 & cofactor metabolic process \\
\hline GO:0044710 & single-organism metabolic process \\
\hline GO:0044281 & small molecule metabolic process \\
\hline GO:0043933 & macromolecular complex subunit organization \\
\hline GO:0065003 & macromolecular complex assembly \\
\hline GO:0051179 & localization \\
\hline GO:0009058 & biosynthetic process \\
\hline GO:0032991 & macromolecular complex \\
\hline GO:0005829 & cytosol \\
\hline GO:0005488 & binding \\
\hline GO:0043167 & ion binding \\
\hline GO:0005515 & protein binding \\
\hline GO:0019899 & enzyme binding \\
\hline GO:0008289 & lipid binding \\
\hline GO:0016491 & oxidoreductase activity \\
\hline GO:0003676 & nucleic acid binding \\
\hline GO:0097159 & organic cyclic compound binding \\
\hline GO:1901363 & heterocyclic compound binding \\
\hline GO:0003824 & catalytic activity \\
\hline
\end{tabular}

Categroy - Biological Process - Cellular Component - Molecular Function 


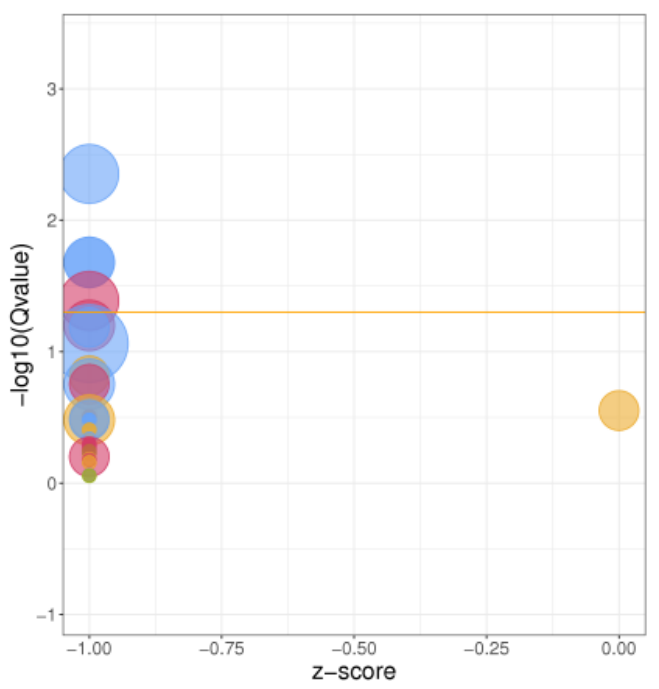

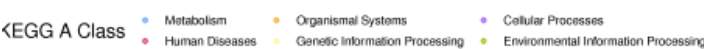

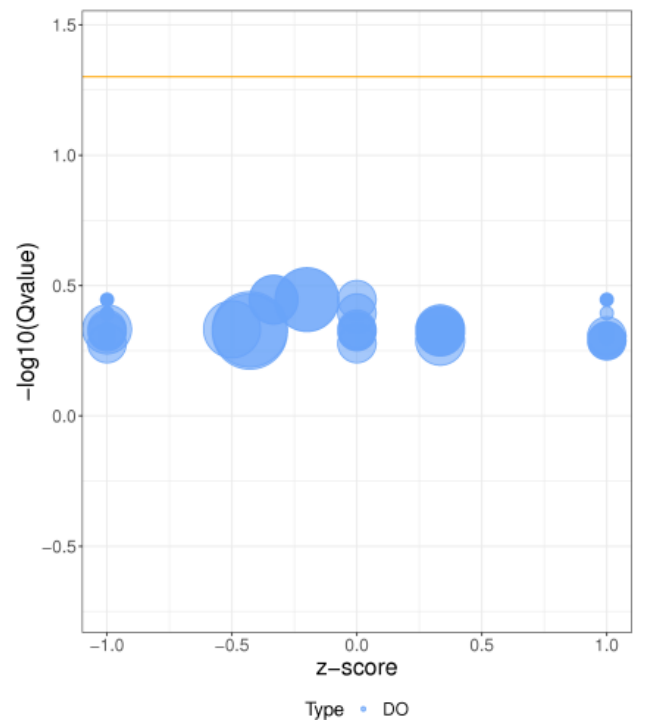

Pathway Top 20

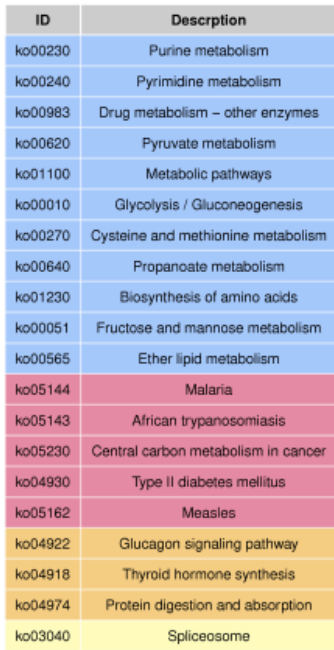

DO term Top 20

\begin{tabular}{|c|c|}
\hline ID & Descrption \\
\hline DOID:1561 & cognitive disorder \\
\hline DOID:2468 & psychotic disorder \\
\hline DO1D:5419 & schizophrenia \\
\hline DO10:6432 & pulmonary hypertension \\
\hline DOID:150 & disease of mental health \\
\hline DOID:12169 & carpal tunnel syndrome \\
\hline DOID:12978 & Plasmodium vivax malaria \\
\hline DOID:13042 & persistent fetal circulation syndrome \\
\hline DOID:1751 & malignant conjunctiva melanoma \\
\hline DOID:1827 & idiopathic generalized eplepsy \\
\hline DO1D-2224 & hemornagic thrombocythemia \\
\hline DO1D:5467 & conjunctival cancer \\
\hline DOID:573 & nerve compression syndrome \\
\hline DO1D:6367 & acral lentiginous melanoma \\
\hline DOID:750 & peptic ulcer disease \\
\hline DO1D:8923 & skin melanoma \\
\hline DO1D:6000 & congestive heart fallure \\
\hline DO1D:0050622 & reproductive organ benign neoplasm \\
\hline DOID:0060086 & female reproductive organ benign neoplasm \\
\hline DOID:0060095 & uterine benign neoplasm \\
\hline
\end{tabular}




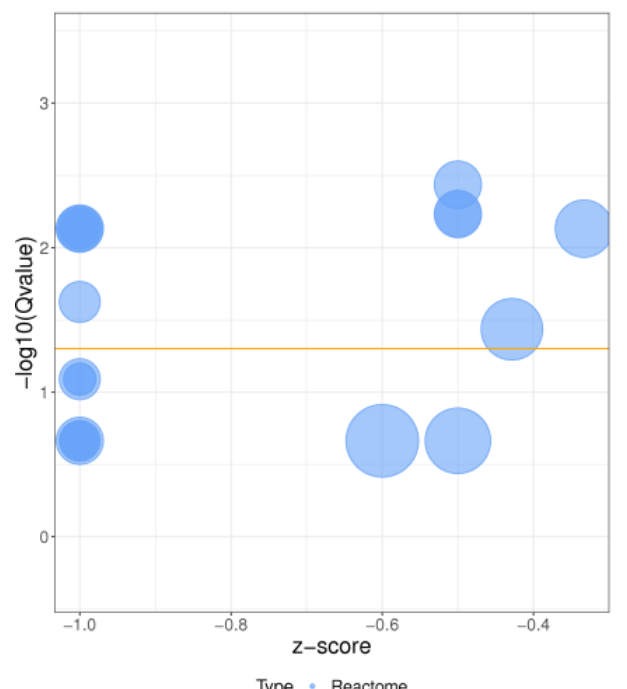

Reactome Top 20

\begin{tabular}{|c|c|}
\hline ID & Descrption \\
\hline A-HSA-2168895 & Haptogobin tinds Hemoglobin \\
\hline R-HSA-2168883 & Heploglobin Hemoglobin binds $\mathrm{CD} 163$ \\
\hline R-HSA-2230938 & Hemoglobin:Haptoglobin:CD163 is endocylosed \\
\hline R-HSA-1237044 & Erytrocytes teks up catbon dioxidg and relaass axyen \\
\hline R-HSA-1247673 & Eytrocytes take up axpen and relasse carbon Gioxida? \\
\hline R-HSA-1480926 & $\mathrm{O} \cdot \mathrm{CO} 2$ exchange in erythrocytes \\
\hline R-HSA-1237325 & $\begin{array}{l}\text { Hemogibbin A As protonated and carbeamated } \\
\text { causing retease of axyen }\end{array}$ \\
\hline R-HSA-1247668 & Hemoglobin A binds arygen and rebaseses prolons and carbon doride \\
\hline A-HSA-6806831 & CYeSAs reduce MeHt to HoA \\
\hline R-HSA-2168880 & Scavenging of heme trom plasma \\
\hline R-HSA-2168884 & Feritherme is transterred from Methemoghbian to Hemopexin \\
\hline R-HSA-2173782 & Binding and Uptetsa of Ligands by Scaverger Recoptors \\
\hline R-HSA-2168839 & Happogocoin-relatad Protein binds Hemogcoin \\
\hline A-HSA-482619 & $\{d \mid N O P+A T P \Leftrightarrow(d) N T P+A D P(N M E 1,2,3)$ \\
\hline A-HSA-482621 & $\{d \mid N T P+$ ADP $\Leftrightarrow\{$ d d NDP + ATP (NME1 2,3$\}$ \\
\hline A-HSA-499943 & 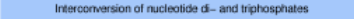 \\
\hline R-HSA-5653656 & Vesicle-modated transport \\
\hline R-HSA-1008220 & $\begin{array}{l}\text { Expression of globin ganes undar control } \\
\text { of the beta ghain control region }\end{array}$ \\
\hline R-HSA-2213205 & $\begin{array}{l}\text { Type XIII and XIV coliagens associato } \\
\text { wheth type I and type II fitrils. }\end{array}$ \\
\hline A-HSA-1430728 & Metaboism \\
\hline
\end{tabular}

